\title{
Diagnósticos de enfermagem identificados em pessoas com diabetes tipo 2 mediante abordagem baseada no Modelo de Orem ${ }^{1}$
}

\author{
Nursing diagnosis identified in people with diabetes type 2 by means of an approach \\ based on Orem's model
}

\section{Diagnósticos de enfermería identificados en personas con diabetes tipo 2 a través de abordaje basado en el modelo de Orem}

\begin{abstract}
Alyne Coelho Moreira Milhomem', Fabiane Fassini Mantelli", Graziela Aparecida Valente Lima'"', Maria Márcia Bachion' ${ }^{\prime V}$, Denize Bouttelet Munari ${ }^{\mathrm{V}}$
\end{abstract}

\section{RESUMO}

Esta pesquisa objetivou descrever alguns fatores condicionantes básicos do autocuidado e analisar os diagnósticos de enfermagem da North American Nursing Diagnosis Association identificados em pessoas com diabetes tipo 2, mediante abordagem baseada na teoria de Orem. Participaram do estudo sete pessoas, avaliadas de maio a junho de 2006. Os fatores condicionantes básicos do autocuidado neste grupo foram: idade entre 46 a 71 anos, predominando o sexo feminino, estado civil casado, ensino médio completo, aposentados, todos com antecedentes familiares de diabetes. Os diagnósticos de enfermagem identificados em todos os participantes foram: controle ineficaz do regime terapêutico; comportamento de busca de saúde; risco para perfusão tissular cardíaca, renal, periférica e/ou cerebral ineficaz; percepção sensorial perturbada (visual), conhecimento deficiente sobre alimentação, cuidado com as extremidades, complicações da doença e prática de exercícios físicos; disposição para conhecimento aumentado acerca do diabetes mellitus tipo 2 e do regime terapêutico; risco de infecção; risco de lesão micro e macrovascular; risco para quedas e risco de integridade da pele prejudicada. Outros dezesseis diagnósticos foram identificados, com uma freqüência menor. Este perfil denota a necessidade de estabelecer um sistema de enfermagem de apoio e orientação sistematizado e efetivo, de modo a aumentar a agência de autocuidado dos participantes.

Palavras chave: Diabetes mellitus tipo 2; Diagnóstico de enfermagem; Teoria de Enfermagem.

\section{ABSTRACT}

This research aimed to describe some of the basic conditioning factors to self-care and analyze the nursing diagnosis of the North American Nursing Diagnosis Association among diabetes type 2 carriers by means of an approach based on Orem's theory. Seven diabetic people took part in this study; they were evaluated during the period of May to June of 2006 . The basic conditioning factors to selfcare in this group were: ages between 46 and 71 , with predominance of the female sex; married people; high-school education; retired people; all with family antecedents for diabetes. The nursing diagnosis identified in all the participants were: ineffective control of the therapeutic regime; health search behavior; risk for ineffective tissue, cardiac, renal, peripheral and/or cerebral perfusion; disturbed sensorial perception (visual); deficient knowledge about food, care with extremities, disease complication and the practice of physical exercise; disposition for increased knowledge about diabetes type 2 and its therapeutic regime; risk of infection; risk of micro and

\footnotetext{
${ }^{1}$ Monografia de Final de Curso, desenvolvida no Núcleo de Estudos e Pesquisas em Saúde Integral, da Faculdade de Enfermagem da Universidade Federal de Goiás.

1 Acadêmica do 5o ano de graduação da Faculdade de Enfermagem da Universidade Federal de Goiás. Goiânia/GO. E-mail: nina milhomem@hotmail.com

"Acadêmica do 5o ano de graduação da Faculdade de Enfermagem da Universidade Federal de Goiás. Goiânia/GO. E-mail: fabinhafassini@hotmail.com

III Acadêmica do 5o ano de graduação da Faculdade de Enfermagem da Universidade Federal de Goiás. Goiânia/GO. E-mail: gravlima@hotmail.com

Iv Enfermeira. Doutora em Enfermagem. Professora Titular da Faculdade de Enfermagem da Universidade Federal de Goiás. Goiânia/GO. E-mail: mbachion@fen.ufg.br.

$\checkmark$ Enfermeira. Doutora em Enfermagem, Professora Titular da Faculdade de Enfermagem da Universidade Federal de Goiás. Goiânia/GO. E-mail: denize@fen.ufg.br
} 
macro vascular damage; risk of falling and risk for skin integrity damage.

Key words: Mellitus diabetes type 2; Nursing diagnosis; Nursing theory.

\section{RESUMEN}

Esta investigación tuvo como objetivos describir algunos factores condicionantes básicos de auto-cuidado y de analizar los diagnósticos de enfermaría de la North American Nursing Diagnosis Association identificados en personas portadoras de diabetes tipo 2 a través de abordaje basado en la teoría de Orem. Participaron del estudio siete personas diabéticas evaluadas durante el período de mayo a junio del 2006. Los factores condicionantes básicos del auto cuidado en este grupo fueron: edad entre 46 a 71 años, predominando el sexo femenino, de estado civil casado, secundaria completa, jubilados, todos

\section{NTRODUÇÃO}

$O$ Diabetes Mellitus (DM) tem sido conceituado de diferentes formas ${ }^{(1)}$, mas no geral prevalece noção de que se trata de uma "síndrome de etiologia múltipla, decorrente da falta de insulina e/ou da incapacidade da insulina de exercer adequadamente seus efeitos. Esta é caracterizada por hiperglicemia crônica freqüentemente acompanhada de dislipidemia, hipertensão arterial e disfunção endotelial"(2). Sua incidência e prevalência já alcançam proporções epidêmicas, consideradas graves, à medida que pode passar despercebido, levando os indivíduos ao risco de complicações crônicas mesmo antes do diagnóstico ${ }^{(3)}$.

Há vários tipos de DM, que diferem na etiologia, evolução clínica e tratamento(4). O mais freqüente é o tipo 2 , que corresponde a aproximadamente $90 \%$ dos $\operatorname{casos}^{(5)}$. Para a manutenção de sua saúde, as pessoas diabéticas precisam adotar práticas estritamente saudáveis, como a alimentação adequada, exercícios físicos regulares, exames médicos periódicos, utilização correta de medicação, além de manejo adequado do estresse, não utilização do fumo e, parcimônia no uso de bebidas alcoólicas ${ }^{(2)}$.

Em nossa experiência de atendimento às pessoas diabéticas percebemos que existem con antecedentes familiares de diabetes. Los diagnósticos de enfermaría que fueron identificados en todos los participantes fueron: control ineficaz del régimen terapéutico, comportamiento de búsqueda de salud, riesgo de perfusión tisular cardíaca, renal, periférica y/o cerebral, ineficaz percepción sensorial perturbada (visual), conocimiento insuficiente sobre alimentación, cuidado con las extremidades, complicaciones de la enfermedad y práctica de ejercicios físicos, disposición para conocimiento aumentando al respecto del diabetes mellitus tipo 2 y del régimen terapéutico, riesgo de infección, riesgo de lesión micro y macro vascular, riesgo de caídas y riesgo de la integridad de la piel perjudicada.

Palabras clave: Diabetes mellitus tipo 2; Diagnóstico de enfermería; Teoría de enfermería.

situações que revelam escolhas nem sempre saudáveis na decisão de práticas de autocuidado. Por outro lado, no atual contexto de atendimento à saúde, as pessoas diabéticas não têm sido abordadas de modo ampliado. São focalizados maciçamente os aspectos biológicos e os níveis glicêmicos, em termos de sua adequacidade ou não, ao invés dos saberes e práticas, habilidades e perspectivas da pessoa diabética sobre a situação de saúde que vivenciam.

Para que $o$ atendimento de enfermagem seja individualizado, resolutivo e de qualidade a estas pessoas e seus familiares é necessário a utilização do processo de enfermagem, que inclui cinco etapas, ou seja, coleta e análise sistemática e organizada dos dados, identificação das necessidades do cliente, que são descritas mediante a utilização de uma taxonomia de diagnósticos de enfermagem, planejamento, implementação e avaliação da assistência. Para identificação e descrição das necessidades das pessoas diabéticas pode ser utilizada a taxonomia de diagnósticos de enfermagem da North American Nursing Diagnosis Association (NANDA) ${ }^{(6)}$, que é compatível com diferentes Modelos ou Teorias de Enfermagem.

Uma teoria de enfermagem que contempla os pressupostos adotados pelo Ministério da 
Saúde e pela Sociedade Brasileira de Diabetes, na abordagem de pessoas com diabetes, é a de Dorothea Orem, cujo foco é o autocuidado, definido como a prática de atividades que os indivíduos realizam para manter a própria vida, saúde e bem estar ${ }^{(7)}$.

Para suprir suas necessidades ou requisitos de autocuidado, os seres humanos precisam dispor ou desenvolver habilidades, que são influenciadas por condicionantes básicos de autocuidado. Trata-se de um conjunto de fatores intrínsecos e extrínsecos do indivíduo que afetam sua capacidade para ocupar-se do seu autocuidado, bem como a qualidade e a quantidade do autocuidado requerido, sendo eles: idade, sexo, orientação sócio-cultural, sistema familiar, padrão de vida, fatores ambientais, acesso a recursos básicos de saúde, estado de desenvolvimento, estado de saúde, fatores do sistema de cuidado de saúde. Cabe ao enfermeiro avaliar a capacidade da pessoa em relação aos requisitos de autocuidado. Em caso de áreas de déficit, são planejados sistemas de enfermagem, que combinam diferentes modos de ajuda ${ }^{(7)}$.

Frente ao exposto, o presente estudo teve como objetivos caracterizar alguns fatores condicionantes básicos do autocuidado e analisar 0 perfil dos diagnósticos de enfermagem da North American Nursing Diagnosis Association (NANDA) $^{(6)}$ identificado junto à pessoas com diabetes, a partir de um roteiro de coleta de dados baseado na teoria de Orem.

Os resultados desta pesquisa poderão subsidiar a compreensão dos fatores que influenciam na capacidade e demandas de autocuidado apresentadas pelas pessoas diabéticas e subsidiar o planejamento de ações para melhoria da qualidade do seu atendimento. O mapa de diagnósticos poderá ser comparado com a avaliação de enfermagem de outros grupos, em outras áreas geográficas, de modo a estabelecer um panorama da situação de saúde dos diabéticos na perspectiva de enfermagem.

\section{METODOLOGI A}

Estudo descritivo realizado de maio a junho de 2006, em uma associação de pessoas com diabetes mellitus, no Estado de Goiás.
Trata-se de instituição filantrópica, sem fins lucrativos. Participam das reuniões mensais, em média, 20 a 30 associados. A população deste estudo envolveu pessoas com diabetes do tipo 2 , de ambos os sexos, de qualquer nível de escolaridade e profissão, independente do tratamento que estavam realizando e que fossem filiados à associação de diabetes onde foi realizada a pesquisa.

Participaram da amostra de conveniência sete pessoas, que atenderam os seguintes critérios de inclusão: ter idade maior ou igual a 18 anos, ser capaz de expressar-se oralmente e de se deslocar até a associação de diabéticos. Os critérios de exclusão adotados foram: o desejo do participante em retirar o consentimento e abandono da pesquisa. Neste contexto, nenhum participante foi excluído.

Este estudo faz parte de um projeto maior, intitulado "Processo de enfermagem baseado no modelo de Orem como estratégia para o atendimento a pessoas com diabetes tipo 2", aprovado pelo Comitê de Ética em Pesquisa Médica Humana e Animal do Hospital das Clínicas da Universidade Federal de Goiás, com o número de protocolo 017/2006.

O projeto foi apresentado em reunião mensal dos associados, ocasião em que formalizamos o convite para participar da pesquisa. Os associados mais assíduos nas atividades da associação foram convidados individualmente, mediante contato telefônico, para uma reunião onde foi reapresentada a proposta de pesquisa e realizada a entrega do Termo de Consentimento Livre e Esclarecido, para aqueles que aceitassem participar.

$\mathrm{Na}$ coleta de dados foi utilizado um roteiro baseado no modelo de Dorothea Orem, que incluiu procedimentos de entrevista, exame físico, aplicação de escala de avaliação cognitiva e dosagem de glicemia capilar, respeitando princípios de bioética e de biossegurança. O roteiro teve duas partes: a primeira, de avaliação dos condicionantes básicos de autocuidado e requisitos de autocuidado de desvio da saúde. A outra parte avaliava as necessidades de autocuidado universal e da agência de autocuidado.

A cada encontro, os dados foram examinados para elaboração de diagnósticos de 
enfermagem da NANDA ${ }^{(6)}$ mediante julgamento clínico, por dois pesquisadores, que discutiam cada caso, sendo que um deles acompanhou todos os casos.

Foi necessário realizar uma média de quatro consultas por participante com, em média, cinqüenta minutos de duração, para obter as informações previstas no protocolo de coleta de dados. Os diagnósticos identificados deram origem ao planejamento dos sistemas de enfermagem pertinentes para atender a cada um dos participantes, o que foi realizado posteriormente, por outra equipe de pesquisadores. A análise dos condicionantes básicos do autocuidado e dos diagnósticos de enfermagem identificados foi realizada mediante procedimentos de estatística descritiva (freqüência simples e percentual).

\section{RESULTADOS E DISCUSSÕES}

Inicialmente foram caracterizados os condicionantes básicos do autocuidado investigados e, a seguir, foram abordados os diagnósticos de enfermagem identificados.

\section{Condicionantes básicos do autocuidado}

Entre os sete participantes do estudo, três eram homens e quatro mulheres. A ocorrência de DM é a mesma em homens e mulheres ${ }^{(5)}$. Apesar disto, notamos que as mulheres procuram mais o serviço de saúde do que os homens, assim como transmitem mais os saberes e práticas acerca da manutenção da saúde e também cuidam da saúde de seus familiares ${ }^{(9)}$. Entre os participantes, as mulheres cuidam de si próprias e de familiares, enquanto que os homens cuidam de si e são cuidados pelas esposas.

A idade interfere na capacidade, no tipo e na quantidade de autocuidado requerido. A faixa etária dos sujeitos variou de 46 a 71 anos de idade, sendo que cinco deles apresentavam idade maior ou igual a 65 anos. Todos eram independentes para as atividades de vida diária, sendo que apenas dois necessitavam de ajuda para as atividades instrumentais de vida diária, mais especificamente, para o transporte e realização de compras, pela falta de veículo próprio e dificuldade de carregar peso, configurando-se como um grupo ativo, com capacidade funcional preservada.

A estrutura da família que reside no mesmo domicílio dos participantes era diversificada, mas em média, cada um deles compartilhava o lar com mais três moradores (filhos, netos, pais, cunhado). Quanto ao estado civil, 05 participantes eram casados, 01 solteiro e 01 viúvo.

O apoio da família é um dos fatores condicionantes do autocuidado, segundo o Modelo de Orem $^{(7)}$. Conforme relato dos participantes, os familiares têm ajudado no controle da doença, especialmente no suporte emocional, apoio para uma alimentação mais saudável, realização de atividades físicas regulares (caminhadas), além de acompanhamento dos resultados de glicemia capilar e monitoramento de estados de hipoglicemia. Uma das participantes não contava com o apoio da família neste sentido, atuando como principal cuidadora dos pais e dos filhos.

A família constitui um sistema no qual o comportamento de cada um dos membros é interdependente ao dos outros. Pode ser um fator facilitador quando a família se torna um ponto de apoio, incentivando o sujeito no comportamento de busca de saúde. Configurase fator dificultador quando a mesma prejudica esta busca de saúde, seja por descaso ou excesso de cuidado ${ }^{(10)}$.

Estudos revelam que as pessoas com diabetes mellitus que tiveram apoio adequado de familiares aderiram melhor às práticas de autocuidado e que avaliar os meios de apoio do paciente pode auxiliar na identificação das necessidades de assistência ${ }^{(10)}$.

Em relação à escolaridade e ocupação, um participante tinha o Ensino Fundamental incompleto, sendo o mesmo aposentado; um participante apresentava Ensino Fundamental completo, sendo o mesmo também aposentado; quatro participantes tinham o Ensino Médio completo, sendo um aposentado, dois do lar e um autônomo; e um apresentava Ensino Superior completo e exercia a profissão de advogado.

Segundo a literatura, o risco de se desenvolver problemas crônicos de saúde é 
Milhomem ACM, Mantelli FF, Lima GAV, Bachion MM, Munari DB.Diagnósticos de enfermagem identificados em pessoas com diabetes tipo 2 mediante abordagem baseada no Modelo de Orem. Revista Eletrônica de Enfermagem [Internet]. 2008; 10(2):321-336. Available from: http://www.fen.ufg. br/revista/v10/n2/v10n2a04.htm

inversamente proporcional ao número de anos de escolaridade formal, sendo que indivíduos com menos de 3 anos de escolaridade apresentam $28 \%$ mais casos de complicações do que aqueles com 11 anos ou mais de escolaridade ${ }^{(12)}$.

Contudo, até o final da coleta de dados, nenhum participante tinha alteração na integridade da pele nos pés, comprometimento da função renal, história de doença cérebro vascular. Três participantes eram também hipertensos arteriais, sendo que um deles tinha história de infarto agudo do miocárdio há alguns anos. Nenhum dos participantes tinha diagnóstico de retinopatia diabética. As complicações relatadas eram do tipo agudas: todos apresentavam hiperglicemia, sendo persistente em 6 casos e esporádica em um caso. Um dos participantes tinha hipoglicemia recorrente. Nos últimos doze meses não sofreram internações e estavam em acompanhamento exclusivamente ambulatorial. A interação com outros fatores condicionantes básicos positivos tais como os recursos ambientais, podem ter levado este grupo a apresentar melhor condição de saúde do que se poderia esperar, em relação às complicações do diabetes.

Outro fator condicionante básico do autocuidado são as experiências anteriores que as pessoas tiveram em relação ao problema de saúde enfrentado (Quadro 1).

Quadro 1: Distribuição dos participantes, segundo antecedentes familiares e modo de descoberta da doença. Goiânia, 2006.

\begin{tabular}{|c|c|c|}
\hline Participante & $\begin{array}{c}\text { Antecedentes } \\
\text { familiares }\end{array}$ & Modo de descoberta \\
\hline $\mathrm{P}_{1}$ & Pais e irmãos & $\begin{array}{c}\text { Eu sentia muita fome, urinava muito e sentia muita sede; minha irmã faleceu } \\
\text { por complicações do diabetes e então procurei o médico porque eu suspeitava } \\
\text { que tivesse diabetes pelos sintomas que eu estava apresentando }\end{array}$ \\
\hline$P_{2}$ & Irmãos & $\begin{array}{c}\text { Três meses antes de saber que eu era diabética, comecei a enxergar como se } \\
\text { tivesse uma nuvem escura tampando minha visão. Fui ao oftalmologista e ele } \\
\text { pediu exames que eu não fiz. Após alguns dias, eu desmaiei e fui levada ao } \\
\text { hospital onde descobri que tinha diabetes }\end{array}$ \\
\hline$P_{3}$ & Tia & $\begin{array}{l}\text { Procurei orientação médica pois sentia muita sede e urinava muito à noite, } \\
\text { assim foi realizado exames e diagnosticado a doença }\end{array}$ \\
\hline $\mathrm{P}_{4}$ & Pais & $\begin{array}{c}\text { Comecei a emagrecer e sentir muita sede. Marquei consulta com um médico. } \\
\text { Eu estava em casa, ia ao médico à tarde. Comecei a passar mal de manhã. Eu } \\
\text { comecei a tremer e desmaiei. Fui levado para o pronto socorro e de lá fui para } \\
\text { a UTI. Estava bem e consciente; eles me levaram só para estabilizar meu } \\
\text { estado. Não dormi durante a noite toda. No outro dia de manhã tive alta da } \\
\text { UTI e recebi o diagnóstico de diabetes }\end{array}$ \\
\hline $\mathrm{P}_{5}$ & Não há & Fui fazer exames de rotina e a minha taxa glicêmica estava alterada, com 161 \\
\hline$P_{6}$ & Mãe & $\begin{array}{c}\text { Apareceu um nódulo na minha perna esquerda, o médico fez uma cirurgia e } \\
\text { retirou, mas como a ferida não cicatrizava eu voltei no médico, ele pediu } \\
\text { exames e então descobri que era diabética }\end{array}$ \\
\hline $\mathrm{P}_{7}$ & $\begin{array}{l}\text { Mãe, tios, avós } \\
\text { e } \\
\text { irmãos }\end{array}$ & $\begin{array}{l}\text { O médico pediu alguns exames porque eu ia fazer uma cirurgia para retirar o } \\
\text { útero, e então eu descobri que a taxa de glicose tava alta. Depois disso, repeti } \\
\text { os exames e continuava alta, então o médico disse que era diabetes. }\end{array}$ \\
\hline
\end{tabular}

Pela lógica, a convivência com pessoas diabéticas na família poderia fazer com que as pessoas interpretassem de modo mais acurado os sinais e sintomas sugestivos desta síndrome. Além disso, seria esperada a adoção de ações para sua detecção precoce. Contudo, no decorrer do estudo foi possível constatar que, embora todos os participantes apresentassem antecedentes familiares de diabetes mellitus tipo 2, isto não foi fator determinante na maioria dos casos para o diagnóstico precoce.

Neste contexto, é importante salientar que na abordagem das pessoas diabéticas pelos profissionais de enfermagem é necessário considerar a dimensão familiar, isto é, que a diabetes pode estar presente em outras pessoas da família, em especial, parentes de primeiro grau, sendo indicadas atividades de promoção da saúde voltadas para estes familiares, mesmo 
que não apresentem a doença. Em relação aos condicionantes analisados, percebemos que se apresentavam predominantemente favoráveis ao desenvolvimento da capacidade do autocuidado.

\section{Diagnósticos de enfermagem identificados}

Identificamos 23 diferentes diagnósticos de enfermagem, variando de 15 a 20 por participante.

A apresentação dos diagnósticos foi realizada segundo os domínios a que pertencem, de acordo com a NANDA, sendo que um domínio representa uma "esfera de atividade, estudo ou interesse"(6).

Dentro do domínio "promoção de saúde"(6) identificamos os diagnósticos de enfermagem controle ineficaz do regime terapêutico e comportamento de busca de saúde que foram encontrados em todos os sete participantes. Os respectivos fatores relacionados e características definidoras destes diagnósticos estão dispostos na Tabela 1.

Tabela 1: Diagnósticos de enfermagem do domínio "promoção de saúde" e respectivos fatores relacionados e características definidoras, identificados em pessoas diabéticas ( $n=7)$. Goiânia, 2006.

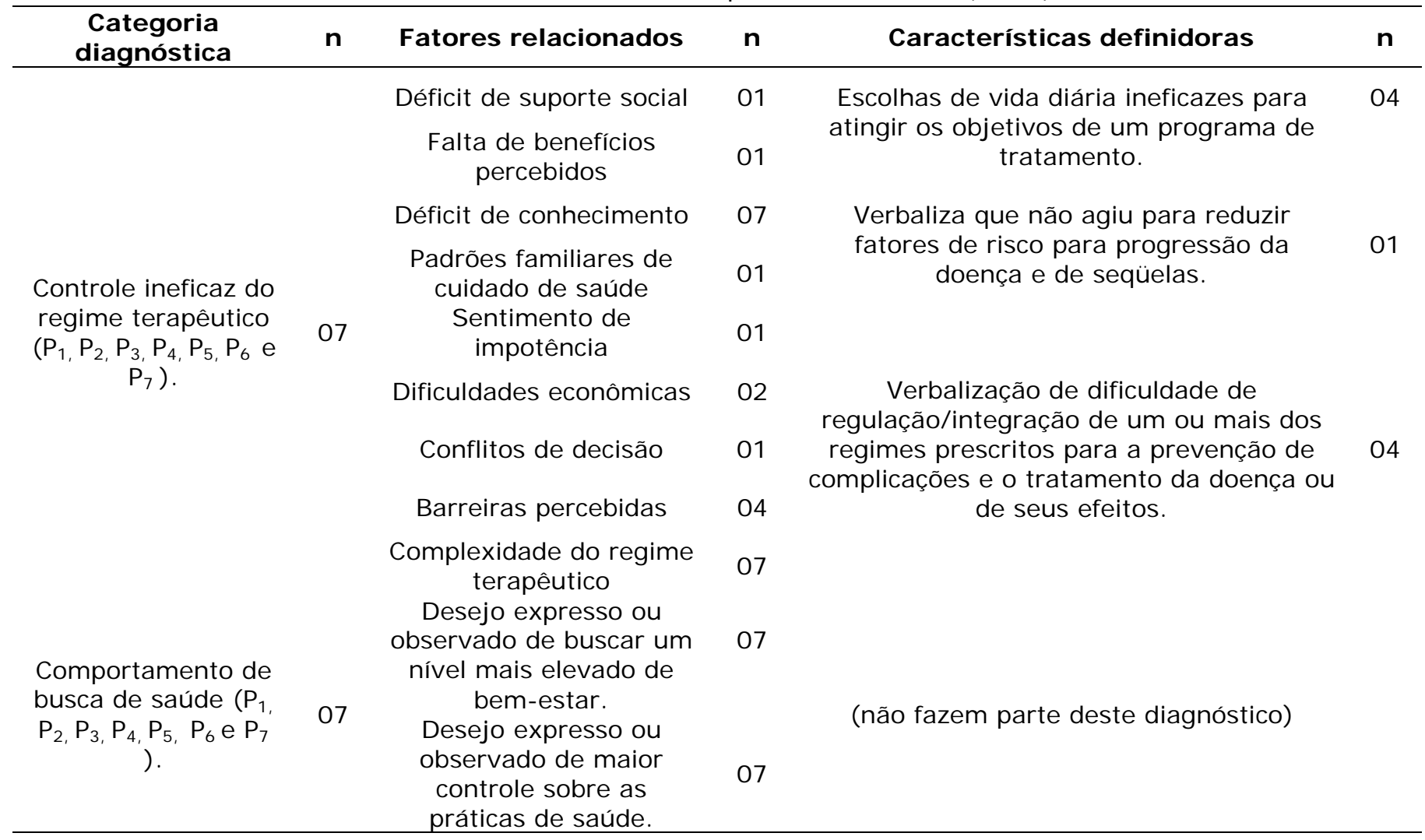

No conjunto, os fatores relacionados para o controle ineficaz do regime terapêutico mostram a influência dos fatores condicionantes básicos ligados ao suporte ambiental, (que corresponde ao apoio social, da família, os recursos financeiros, entre outros), além de experiências nem sempre bem sucedidas de alcance de benefícios percebidos, por falta de habilidades e falta de conhecimento.

$O$ apoio social se define como sendo qualquer informação, falada ou não, e/ou auxílio material oferecido por pessoas que se conhecem e que resultam em efeitos emocionais e comportamentos positivos. O apoio social melhora a saúde e o bem-estar das pessoas, atuando em situações como um fator de proteção, e como uma ferramenta de autonomia para os indivíduos, na medida em que estes aprendem e compartilham modos de lidar com o processo saúde - doença ${ }^{(11)}$. Isso quer dizer que para haver o controle eficaz do regime terapêutico é necessário que a pessoa com diabetes disponha de suporte dos amigos, familiares e/ou profissionais de saúde que os incentive e os apóie na manutenção da saúde; que tenham acesso a serviços/ pessoas 
dispostas a ajudá-los a obter conhecimentos acerca da doença e que possibilitem que as pessoas diabéticas se sintam capazes de realizar o autocuidado de maneira satisfatória e que se sintam motivados a se engajar de maneira positiva no regime terapêutico, uma vez que este é complexo e requer mudanças nos hábitos diários de vida e novas práticas de saúde.

Durante a coleta de dados, a maioria dos participantes citou que fez escolhas de vida diária que não foram condizentes com o tratamento e que tiveram dificuldades em realizar e/ou manter práticas de saúde adequadas relativas ao tratamento e prevenção de complicações.

Considerando as evidências disponíveis, que são suficientes para afirmar que o melhor controle da glicemia, da pressão arterial e da dislipidemia resulta em uma redução significativa nas complicações microvasculares e macrovasculares da pessoa com diabetes ${ }^{(3)}$, reafirmamos a necessidade de buscar estratégias de intervenção profissional com vistas a melhorar a eficácia do controle do regime terapêutico, para prevenir ou retardar o aparecimento das complicações crônicas e assim favorecer a qualidade de vida de pacientes e seus familiares ${ }^{(10)}$.

Nesse sentido, assume especial importância o diagnóstico de enfermagem comportamento de busca de saúde que é definido como a "busca ativa de caminhos para alterar hábitos pessoais de saúde e /ou o ambiente a fim de mover-se rumo a um nível mais elevado de saúde"(6). Todos os participantes demonstraram desejo de buscar um nível mais elevado de bem-estar e de maior controle sobre as práticas de saúde.

A presença simultânea destes dois diagnósticos no domínio "promoção da saúde" pode significar que o controle eficaz do regime terapêutico exige das pessoas diabéticas um engajamento permanente, embora o suporte do sistema de saúde, a rede de apoio social e a ajuda dos profissionais têm sido insuficientes para garantir a sustentação de comportamentos e escolhas saudáveis.

No domínio "nutrição"(6), foram identificados entre os participantes os diagnósticos de enfermagem nutrição desequilibrada: mais do que as necessidades corporais e volume excessivo de líquidos, como pode ser observado na Tabela 2.

Tabela 2: Diagnósticos de enfermagem segundo o domínio "nutrição", fatores relacionados e características definidoras, Goiânia, 2006.

\begin{tabular}{|c|c|c|c|c|c|}
\hline Categoria diagnóstica & $\mathbf{n}$ & Fatores relacionados & $\mathbf{n}$ & $\begin{array}{l}\text { Características } \\
\text { definidoras }\end{array}$ & $\mathbf{n}$ \\
\hline $\begin{array}{l}\text { Nutrição desequilibrada: mais do } \\
\text { que as necessidades corporais } \\
\qquad\left(P_{1}, P_{2}, P_{3}, P_{4}, P_{5} \text { e } P_{6}\right)\end{array}$ & 06 & $\begin{array}{c}\text { Ingestão excessiva em relação às } \\
\text { necessidades metabólicas } \\
\text { (carboidratos e/ou gorduras). }\end{array}$ & 06 & $\begin{array}{l}\text { IMC de sobrepeso. } \\
\text { Comer em resposta a } \\
\text { estímulos internos } \\
\text { (ansiedade). } \\
\text { Padrão de alimentação } \\
\text { disfuncional relatado. }\end{array}$ & 04 \\
\hline Volume excessivo de líquidos ( $P_{2}$ & 01 & $\begin{array}{l}\text { Mecanismos reguladores } \\
\text { comprometidos. }\end{array}$ & 01 & Edema. & 01 \\
\hline
\end{tabular}

No diagnóstico de enfermagem nutrição desequilibrada: mais do que as necessidades corporais encontramos como fator relacionado a ingestão excessiva em relação às necessidades metabólicas (carboidratos e/ou gorduras) e as seguintes características definidoras: IMC de sobrepeso, comer em resposta a estímulos internos (ansiedade), padrão de alimentação disfuncional relatado.
Segundo relatório da Organização Mundial da Saúde (OMS) sobre dieta, nutrição e prevenção de doenças crônicas nãotransmissíveis, a associação entre o ganho de peso, obesidade abdominal, sedentarismo e o desenvolvimento de diabetes mellitus tipo 2 é convincente, e o consumo alimentar habitual é considerado um dos principais fatores passíveis 
Milhomem ACM, Mantelli FF, Lima GAV, Bachion MM, Munari DB.Diagnósticos de enfermagem identificados em pessoas com diabetes tipo 2 mediante abordagem baseada no Modelo de Orem. Revista Eletrônica de Enfermagem [Internet]. 2008; 10(2):321-336. Available from: http://www.fen.ufg. br/revista/v10/n2/v10n2a04.htm

de modificação relacionados ao desenvolvimento destas doenças ${ }^{(13)}$.

A literatura tem apontado um baixo seguimento dos pacientes à dieta recomendada. $\mathrm{O}$ ato de comer é bastante complexo e envolve uma amplitude de emoções e sentimentos, além de valores culturais específicos. 0 comportamento alimentar está relacionado tanto com aspectos técnicos e objetivos (o que, quanto e onde comemos), como também com aspectos socioculturais e psicológicos ${ }^{(9)}$. Além disso, os idosos portadores de problema crônico de saúde, como o diabetes tipo 2, apresentam maior chance de obesidade ${ }^{(13)}$.

No diagnóstico de enfermagem volume excessivo de líquidos (uma participante), o fator relacionado encontrado foi, mecanismos reguladores comprometidos e a característica definidora observada foi a presença de edema. Esta participante também apresentava hipertensão arterial sistêmica, sem história de comprometimento renal.

Considera-se hipertensão em portadores de diabetes quando a pressão sistólica for igual ou maior que $130 \mathrm{mmHg}$ e a pressão diastólica for igual ou maior que $85 \mathrm{mmHg}$. A hipertensão pode contribuir no desenvolvimento $e$ progressão da aterosclerose de grandes vasos, e agravar a nefropatia e retinopatia diabéticas ${ }^{(5)}$.

A filtração renal eficiente depende de um fluxo sanguíneo adequado, que mantém uma pressão consistente através do glomérulo que é responsável pela filtração. Muitos fatores podem alterar esse fluxo sanguíneo, tais como a hipotensão, pressão aumentada nos túbulos renais, entre outras ${ }^{(15)}$. Assim, os mecanismos reguladores envolvidos neste processo, para esta participante, não puderam ser especificados, necessitando de uma análise mais minuciosa.

No domínio “atividade/repouso"(6) identificamos os seguintes diagnósticos de enfermagem: intolerância a atividade; risco para perfusão tissular cardíaca e/ ou cerebral ineficaz e déficit no autocuidado com as unhas dos pés (Tabela 3 ).

Tabela 3: Diagnósticos de enfermagem segundo o domínio "atividade/repouso", fatores relacionados e características definidoras, Goiânia, 2006.

\begin{tabular}{|c|c|c|c|c|c|}
\hline Categoria diagnóstica & $\mathbf{n}$ & Fatores relacionados & $\mathbf{n}$ & $\begin{array}{c}\text { Características } \\
\text { definidoras }\end{array}$ & $\mathbf{n}$ \\
\hline \multirow[b]{2}{*}{ Intolerância à atividade $\left(P_{2}\right)$} & \multirow[b]{2}{*}{01} & Dor. & 01 & \multirow{5}{*}{$\begin{array}{c}\text { Relato verbal (dor e falta } \\
\text { de ar). } \\
\text { Desconforto (respiratório } \\
\text { desencadeado por } \\
\text { atividade). }\end{array}$} & 01 \\
\hline & & $\begin{array}{c}\text { Desequilíbrio entre a } \\
\text { demanda e oferta de } 02 \text {. }\end{array}$ & 01 & & 01 \\
\hline & & Diabetes mellitus tipo 2 & 07 & & \\
\hline $\begin{array}{l}\text { Risco para perfusão tissular cardíaca, } \\
\text { renal, periférica e/ou cerebral }\end{array}$ & 07 & $\begin{array}{l}\text { Circunferência } \\
\text { abdominal aumentada. }\end{array}$ & 05 & & \\
\hline ineficaz. $\left(P_{1}, P_{2}, P_{3}, P_{4}, P_{5}, P_{6}, P_{7}\right)$ & & $\begin{array}{l}\text { Hipertensão arterial } \\
\text { sistêmica. }\end{array}$ & 04 & & \\
\hline $\begin{array}{l}\text { Déficit no autocuidado com as unhas } \\
\text { dos pés. }\left(P_{2}\right)\end{array}$ & 01 & $\begin{array}{l}\text { Mobilidade prejudicada. } \\
\text { (abdome volumoso). }\end{array}$ & 01 & $\begin{array}{c}\text { Incapacidade de cortar as } \\
\text { unhas do pé. }\end{array}$ & 01 \\
\hline
\end{tabular}

No início da pesquisa, cinco participantes realizavam exercícios físicos regulares, sendo mais freqüente caminhada ao ar livre. Dois não realizavam tais atividades, sendo um deles devido à intolerância à atividade e um outro por falta de motivação.

Os exercícios regulares ajudam a diminuir e manter o peso corporal, a reduzir a necessidade de hipoglicemiantes orais, a diminuir a resistência à insulina e contribuem para uma melhora do controle glicêmico, reduzindo o risco de complicações. No entanto, tais atividades devem ser realizadas após uma avaliação médica minuciosa, que analise as condições macrovasculares e microvasculares. O programa de exercícios sempre deve ser feito sob orientação e controle da equipe de saúde, face aos possíveis riscos ${ }^{(10)}$.

O diagnóstico de enfermagem risco para perfusão tissular cardíaca, renal, periférica e/ ou cerebral ineficaz foi afirmado, considerando-se o fato da macroangiopatia ser uma das complicações crônicas do diabetes mellitus, e engloba a cardiopatia isquêmica, 
doença cerebrovascular e doença vascular periférica $^{(4)}$. O diabetes tipo 2 sabidamente associa-se a vários fatores de risco cardiovasculares, incluindo hipertensão arterial sistêmica, obesidade, resistência à insulina, microalbuminúria e anormalidades nos lipídios e lipoproteínas plasmáticas ${ }^{(3)}$. As complicações macrovasculares do diabetes resultam de alterações nas paredes dos vasos sangüíneos, que sofrem espessamento, esclerose e se tornam ocluídos pelas placas. Essas alterações ateroscleróticas são indistinguíveis das alterações ateroscleróticas nas pessoas não portadoras do diabetes, mas tendem a ocorrer com maior freqüência e em idade mais precoce no diabetes ${ }^{(15)}$.

Apenas um dos participantes apresentou o diagnóstico de enfermagem déficit no autocuidado com as unhas dos pés, sendo a causa a dificuldade de acessar esta parte do corpo, em função do abdome volumoso.

Quando a pessoa não consegue acessar visualmente seus pés, é necessário que uma agência de autocuidado dependente nesse sentido seja identificada pela equipe de saúde e adequadamente preparada para exercer esta atividade.

No domínio "percepção/cognição", definido como "sistema humano de processamento de informações, incluindo atenção, orientação, sensação, percepção, cognição e comunicação"(6) identificamos, conforme mostra a Tabela 4, os diagnósticos de enfermagem percepção sensorial perturbada (visual, auditiva, gustativa e tátil), conhecimento deficiente (sobre alimentação, cuidado com as extremidades, complicações da doença, sinais e sintomas da doença, entre outros).

Tabela 4: Diagnósticos de enfermagem segundo o domínio "percepção/cognição", fatores relacionados e características definidoras, Goiânia, 2006.

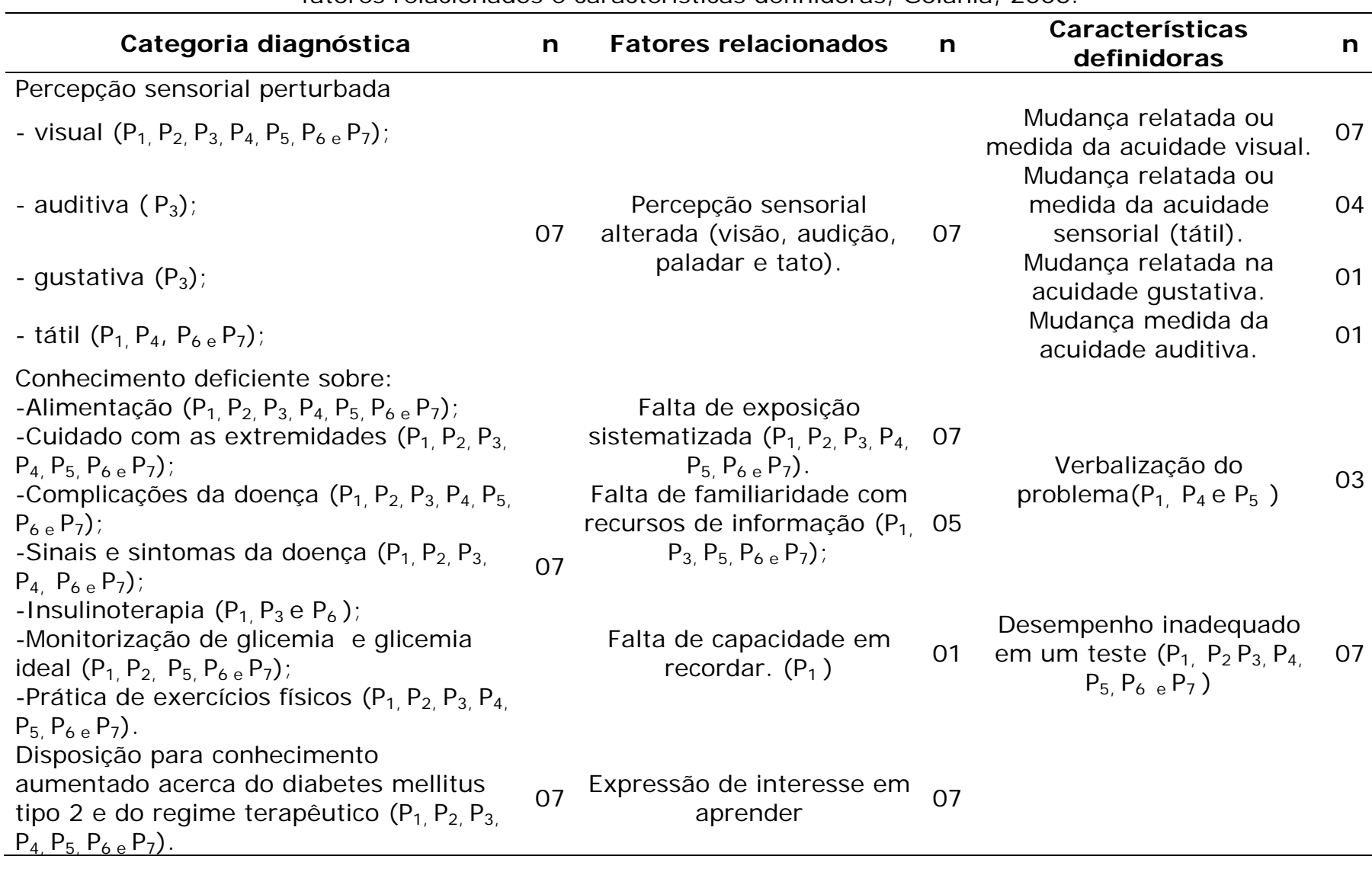

A percepção sensorial visual e a auditiva podem estar perturbadas nos participantes devido tanto ao processo de envelhecimento como pelas complicações do diabetes. Com o processo de envelhecimento ocorre a presbiopia, causada por dificuldade de acomodação do cristalino devido a diminuição da sua elasticidade em idosos. No processo de envelhecimento pode ocorrer ainda a disacusia, decorrente de alterações causadas pelo 
envelhecimento, como a maior probabilidade de impactação do cerúmen, atrofia da membrana timpânica e degeneração das células na base da cóclea(15).

Por outro lado, é importante ressaltar que uma das complicações do diabetes mellitus é a lesão microvascular, que leva à retinopatia. Trata-se de uma das complicações mais comuns e está presente especialmente em pacientes com longo tempo de doença e mau controle glicêmico. A fisiopatologia das alterações microvasculares do tecido retiniano está relacionada à hiperglicemia crônica(16).

A neuropatia diabética apresenta-se sob várias formas, sendo entre as manifestações clínicas da neuropatia periferia está a diminuição da sensibilidade tátil ${ }^{(3)}$.

A detecção precoce do "pé em risco" pode ser feita facilmente pela inspeção e avaliação da sensibilidade através de testes simples e de baixo custo. As recomendações do "Consensus Development on Diabetic Foot Wound Care"(4) são taxativas em determinar que a avaliação da sensibilidade periférica deve fazer parte da rotina do exame físico dos pacientes. O emprego dessas medidas e a educação de profissionais, pacientes e familiares podem reduzir o risco de amputação.

O conhecimento deficiente sobre: alimentação, cuidado com as extremidades, complicações da doença, prática de exercícios de forma correta foi identificado em todos os participantes (Tabela 4). Outros temas também careciam de conhecimento adequado, para levar à independência no autocuidado, tais como sinais e sintomas da doença (06 participantes), monitorização de glicemia e glicemia ideal (05 participantes), insulinoterapia participantes). Lembramos que conhecimento deficiente não significa ausente, mas sim em quantidade e qualidade precária, que não é capaz de sustentar a tomada de decisões e a realização de ações de forma segura.

Os fatores relacionados referidos foram especialmente à falta de exposição sistematizada, desempenho inadequado em um teste (todos os participantes) e à falta de familiaridade com os recursos de informação (05 participantes).

Por outro lado, todos apresentaram o diagnóstico de disposição para conhecimento aumentado acerca do diabetes mellitus tipo 2 e do regime terapêutico relacionado a expressão de interesse em aprender (Tabela 4).

O conhecimento adequado para o paciente é fundamental para a adesão ao tratamento e o manejo da doença. Os profissionais devem entender que a ação educativa é mais do que meramente apresentar uma lista de ações a serem desenvolvidas, na forma de comportamentos prescritos.

As Diretrizes da Educação para a Saúde definem Educação em saúde como "uma atividade planejada que objetiva criar condições para produzir as mudanças de comportamento desejadas em relação à saúde". A ação educativa deve ser abranger percepções, valores, representações simbólicas, relações de poder, crenças e sentimentos, das pessoas envolvidas, não devendo ser concebida como a mera aquisição de novos conhecimentos ${ }^{(17)}$.

Conforme a Tabela 5, a pontuação total obtida pelos participantes no teste de minimental $^{(18)}$ variou entre 23 e 30 pontos, sendo que três participantes atingiram 0 escore máximo de 30 pontos, um participante atingiu escore de 29 pontos, um participante atingiu escore de 28 pontos, um participante atingiu escore de 26 pontos e um participante atingiu escore de 23 pontos. Nenhum participante obteve o escore considerado baixo (20 pontos).

Tabela 5: Distribuição dos participantes segundo pontuação no teste Mini - mental. Goiânia, 2006.

\begin{tabular}{ccccccc}
\hline Participante/ & \multicolumn{5}{c}{ Pontuação em cada item } & \multirow{2}{*}{ Pontuação Total } \\
\cline { 2 - 5 } Idade & Orientação & Memória & Atenção & Lembrança & Linguagem & 30 \\
C1/65 anos & 10 & 03 & 05 & 03 & 09 & 09 \\
C2/68 anos & 08 & 03 & 05 & 01 & 09 & 26 \\
C3/62 anos & 10 & 03 & 05 & 02 & 03 & 30 \\
C4/68 anos & 10 & 03 & 05 & 02 & 03 & 23 \\
C5/71 anos & 10 & 03 & 05 & 03 & 09 & 30 \\
C6/53 anos & 10 & 03 & 05 & 03 & 09 & 28 \\
C7/46 anos & 09 & 03 & 04 & & & \\
\hline
\end{tabular}


Não se pode afirmar, portanto, que o conhecimento deficiente apresentado pelos participantes fosse devido a obstáculos à cognição, já que nenhum deles teve desempenho crítico no teste. Pode ser que o atual modelo de ações desenvolvido nas instituições de saúde seja inadequado para promover uma aprendizagem significativa.

No domínio "sexualidade", foi identificado o diagnóstico de enfermagem disfunção sexual, definido como "mudança na função sexual, que é vista como insatisfatória, nãocompensadora e inadequada"(6). O fator relacionado encontrado foi estrutura corporal da função alterada; e as características definidoras foram verbalização do problema, incapacidade de alcançar a satisfação desejada, alteração no alcance da satisfação sexual, alterações no desempenho do papel sexual percebido e limitações reais ou percebidas pela doença e/ou terapêutica (Tabela 6).

Tabela 6: Diagnósticos de enfermagem segundo o domínio "sexualidade", fatores relacionados e características definidoras, Goiânia, 2006.

\begin{tabular}{|c|c|c|c|c|c|}
\hline $\begin{array}{c}\text { Categoria } \\
\text { diagnóstica }\end{array}$ & $\mathbf{n}$ & Fatores relacionados & $\mathbf{n}$ & Características definidoras & $\mathbf{n}$ \\
\hline $\begin{array}{l}\text { Disfunção sexual }\left(P_{1}\right. \\
\left.\qquad P_{2}, P_{3}, P_{4} \text { e } P_{5}\right)\end{array}$ & 05 & $\begin{array}{l}\text { Estrutura corporal da função } \\
\text { alterada (doença e medicação). }\end{array}$ & 05 & $\begin{array}{c}\text { Verbalização do problema. } \\
\text { Incapacidade de alcançar a satisfação } \\
\text { desejada. } \\
\text { Alteração no alcance da satisfação } \\
\text { sexual. } \\
\text { Alterações no desempenho do papel } \\
\text { sexual percebido. } \\
\text { Limitações reais ou percebidas } \\
\text { impostas pela doença e/ou } \\
\text { terapêutica. }\end{array}$ & $\begin{array}{l}02 \\
03\end{array}$ \\
\hline
\end{tabular}

A sexualidade é uma dimensão importante do ser humano, incluída entre as necessidades humanas básicas, sendo essencial no atendimento holístico. Trata-se de uma questão relacionada tanto aos aspectos orgânicos, quanto aos fatores biopsicossociais. As disfunções sexuais representam distúrbios da resposta sexual. Eles envolvem os distúrbios do desejo, excitação e orgasmo ${ }^{(19)}$.

Muitos fatores, incluindo as mudanças fisiológicas já esperadas e decorrentes do processo de envelhecimento, disfunções induzidas por medicamentos e alterações vasculares associadas com fatores de risco (por exemplo, diabetes, hipertensão e dislipidemias),

Tabela 7: Diagnósticos de enfermagem segundo o domínio "enfrentamento/tolerância ao estresse", fatores relacionados e características definidoras, Goiânia, 2006.

\begin{tabular}{|c|c|c|c|c|c|}
\hline $\begin{array}{c}\text { Categoria } \\
\text { diagnóstica }\end{array}$ & $\mathbf{n}$ & Fatores relacionados & $\mathbf{n}$ & Características definidoras & $\mathbf{n}$ \\
\hline $\begin{array}{c}\text { Adaptação } \\
\text { prejudicada }\left(\mathrm{P}_{6}\right)\end{array}$ & 01 & $\begin{array}{l}\text { Falta de motivação para mudar } \\
\text { comportamentos. } \\
\text { Ausência de suporte social para } \\
\text { mudar práticas. } \\
\text { Estado emocional intenso. } \\
\text { Mudanças no estado de saúde que } \\
\text { requer transformação no estilo de } \\
\text { vida. }\end{array}$ & $\begin{array}{l}01 \\
01 \\
01 \\
01\end{array}$ & $\begin{array}{c}\text { Falha em conseguir um senso de } \\
\text { controle eficiente. } \\
\text { Falha em realizar ações que } \\
\text { preveniriam outros problemas de } \\
\text { saúde. }\end{array}$ & 01 \\
\hline
\end{tabular}

podem influenciar a vida sexual dos indivíduos ${ }^{(3)}$.

No domínio "enfrentamento/tolerância ao estresse"(6), identificamos os diagnósticos de adaptação prejudicada; ansiedade; enfrentamento familiar comprometido e enfrentamento ineficaz (Tabela 7). 


\section{Mudanças no estado de saúde que requer transformação no estilo de vida. \\ Mudanças no estado de saúde que requer transformação no estilo de vida.}

\section{Estresse.}

Ansiedade $\left(P_{3}\right.$ e $\left.P_{6}\right)$.

02

Necessidades não satisfeitas.

Enfrentamento familiar comprometido $\left(\mathrm{P}_{3}\right)$

Enfrentamento ineficaz $\left(P_{5}\right)$
I nformação ou compreensão 01 inadequada ou incorreta por parte de uma pessoa fundamental.

01 Nível inadequado de percepção de
01

01

Inquietação.

01

Sentimento de inadequação. 01 inadequada.
Estar ansioso.

Olhar em torno.

Preocupação expressa.

Ruminação.

Dificuldade para concentrar-se.

Pessoa significativa tenta

01

comportamentos assistenciais ou

de apoio com resultados insatisfatórios.

Resolução de problemas

01

01

01

01

01

01

01 controle.

que os relatos denotam a sensação de ansiedade como uma constante, no dia-a-dia, quase como uma atitude.

Esta situação de tensão emocional pode afetar negativamente no controle do diabetes ${ }^{(6)}$.

Também foram identificado neste domínio, o diagnóstico enfrentamento familiar comprometido, relacionado à informação ou compreensão inadequada ou incorreta por parte de uma pessoa fundamental, associado à pessoa significativa tentar comportamentos assistenciais ou de apoio com resultados insatisfatórios, (1 participante); e enfrentamento ineficaz relacionado a nível inadequado de percepção de controle associado a resolução de problemas inadequada (1 participante).

Sabe-se que a família e os amigos influenciam tanto no controle da doença como no seguimento do tratamento, da dieta e na participação em um programa regular de exercícios. A doença na família afeta tanto o relacionamento entre o casal, entre os irmãos, bem como as relações estabelecidas com o sistema de saúde ${ }^{(10)}$. Assumindo a família como um sistema, fica claro que a experiência de cada um de seus membros afeta a totalidade da dinâmica familiar, dado que o comportamento de cada pessoa afeta e é afetado pelo comportamento de cada uma das outras pessoas. Nessa perspectiva, a enfermagem deve focalizar interações entre seus membros, bem como as interações com os outros subsistemas (profissionais de saúde, parentes, 
Milhomem ACM, Mantelli FF, Lima GAV, Bachion MM, Munari DB.Diagnósticos de enfermagem identificados em pessoas com diabetes tipo 2 mediante abordagem baseada no Modelo de Orem. Revista Eletrônica de Enfermagem [Internet]. 2008; 10(2):321-336. Available from: http://www.fen.ufg. br/revista/v10/n2/v10n2a04.htm

amigos) ao invés de estudar só a pessoa doente ${ }^{(10)}$.

No domínio "segurança/proteção"(6) foram encontrados os diagnósticos de enfermagem risco de infecção, risco de lesão micro e macro vascular, risco para quedas e risco de integridade da pele prejudicada (Tabela 8).

Tabela 8: Diagnósticos de enfermagem segundo o domínio "segurança/proteção", fatores relacionados e características definidoras, Goiânia, 2006.

\begin{tabular}{|c|c|c|c|c|c|}
\hline Categoria diagnóstica & $\mathbf{n}$ & Fatores relacionados & $\mathbf{n}$ & $\begin{array}{c}\text { Características } \\
\text { definidoras }\end{array}$ & $\mathbf{n}$ \\
\hline \multirow{2}{*}{$\begin{array}{l}\text { Risco de infecção }\left(P_{1}, P_{2}, P_{3}, P_{4}\right. \\
\left.P_{5}, P_{6} \text { e } P_{7}\right) \text {. }\end{array}$} & \multirow[b]{2}{*}{07} & \multirow{2}{*}{$\begin{array}{l}\text { Doença crônica. } \\
\text { Procedimento invasivo } \\
\text { (insulinoterapia). }\end{array}$} & 07 & & \\
\hline & & & 04 & & \\
\hline \multirow{3}{*}{$\begin{array}{l}\text { Risco de lesão: } \\
\text { - micro e macrovascular - }\left(P_{1}\right. \\
\left.P_{2}, P_{3}, P_{4}, P_{5}, P_{6} \text { e } P_{7}\right) \\
\text { - tímpano }\left(P_{4}\right)\end{array}$} & \multirow{3}{*}{$\begin{array}{l}07 \\
01\end{array}$} & & & & \\
\hline & & $\begin{array}{l}\text { Perfil sanguíneo anormal. } \\
\text { (hiperglicemia) }\end{array}$ & 07 & & \\
\hline & & $\begin{array}{l}\text { Agente químico (álcool) } \\
\text { Idade maior que } 65 \text { anos. }\end{array}$ & $\begin{array}{l}01 \\
05\end{array}$ & & \\
\hline \multirow{2}{*}{$\begin{array}{l}\text { Risco para quedas }\left(P_{1}, P_{2}, P_{3}\right. \\
\left.P_{4}, P_{5}, P_{6} \text { e } P_{7}\right)\end{array}$} & \multirow[t]{2}{*}{07} & $\begin{array}{l}\text { Agentes anti-hipertensivos. } \\
\text { Inibidores da ECA. }\end{array}$ & $\begin{array}{l}02 \\
02\end{array}$ & & \\
\hline & & $\begin{array}{c}\text { Mudança na glicemia pós- } \\
\text { prandial. }\end{array}$ & 07 & & \\
\hline \multirow[t]{2}{*}{$\begin{array}{l}\text { Risco de integridade da pele } \\
\text { prejudicada }\left(P_{1}, P_{2}, P_{3}, P_{4}, P_{5}, P_{6}\right. \\
\left.\text { e } P_{7}\right) \text {. }\end{array}$} & 07 & $\begin{array}{c}\text { Alterações metabólicas. } \\
\text { Fatores mecânicos (uso de } \\
\text { instrumento cortante na } \\
\text { presença de deficiência } \\
\text { visual). }\end{array}$ & 07 & & \\
\hline & & Sensibilidade tátil alterada. & 04 & & \\
\hline Dentição prejudicada $\left(P_{1}\right.$ e $\left.P_{5}\right)$. & 02 & Barreiras culturais. & 02 & $\begin{array}{l}\text { Perda dos dentes. } \\
\text { Dor. } \\
\text { Presença de placas. }\end{array}$ & $\begin{array}{l}02 \\
01 \\
01\end{array}$ \\
\hline $\begin{array}{l}\text { Proteção ineficaz }\left(P_{4}, P_{5}, P_{6}\right. \\
\left.P_{7}\right)\end{array}$ & 04 & $\begin{array}{c}\text { Perfis sanguíneos anormais } \\
\text { (hiperglicemia). }\end{array}$ & 04 & $\begin{array}{l}\text { Prejuízos na cicatrização. } \\
\text { Alteração neurossensorial }\end{array}$ & $\begin{array}{l}04 \\
01\end{array}$ \\
\hline
\end{tabular}

A infecção é definida como "a condição em que o hospedeiro interage fisiológica e imunologicamente com um microorganismo", e tem como elementos necessários: agente etiológico, condições ambientais, porta de saída, meios de transmissão, porta de entrada e hospedeiro suscetível ${ }^{(15)}$. A pessoa diabética é um hospedeiro suscetível devido a seus perfis sangüíneos anormais. A insulinoterapia, neste caso, é considerada importante porta de entrada devido à freqüente reutilização das seringas descartáveis ${ }^{(10)}$. A falta de informação e treinamento adequado dos pacientes para a auto-aplicação pode ser uma das causas de infecção por insulinoterapia.

Pessoas diabéticas têm dificuldade de cicatrização em áreas com alteração na circulação sangüínea devido às complicações micro e macrovasculares. Nos casos mal controlados, há maior tendência a infecções, sendo necessário um plano de avaliação periódica e sistemática para detectá-las e tratálas o mais precocemente possível ${ }^{(5)}$.
A queda é uma fonte comum e prevenível de mortalidade e morbidade em adultos idosos. A fratura mais comum, a partir de uma queda, é a de quadril, resultante das co-morbidades combinadas da osteoporose e do distúrbio ou situação que provocou a queda. Uma destas situações, no caso do diabetes, pode ser a hipoglicemia. No caso de uso de medicação, os anti-hipertensivos e os inibidores da ECA, pela probabilidade de provocar hipotensão, também são fatores de risco para quedas ${ }^{(15)}$.

A integridade da pele em diabético corre o risco de estar prejudicada devido às alterações na microcirculação. Qualquer distúrbio que reduza a circulação e a nutrição do tecido subcutâneo aumenta o risco de desenvolvimento de lesão de pele ${ }^{(15)}$.

O indivíduo diabético pode experimentar distúrbios na cavidade bucal, sendo a doença periodontal a mais freqüente, podendo levar a perdas dentárias. A cárie pode deteriorar o controle metabólico, agravando, assim, outras complicações crônicas diabéticas ${ }^{(20)}$. 
O acesso aos serviços odontológicos, juntamente com a orientação constante da equipe de saúde, são recursos importantes na busca de suporte para a manutenção da autonomia e uma melhora no quadro geral de saúde do individuo ${ }^{(11)}$.

No domínio "conforto"(6) identificamos em um participante o diagnóstico de enfermagem dor crônica, relacionado a agente lesivo, associado a relato verbal, posição antálgica para evitar a dor e evidência observada. Tratava-se de uma pessoa que havia sofrido traumatismos ósseos múltiplos, havia muitos anos, e que enfrentava dor nesses locais, com períodos de melhora e de exacerbação (Tabela 9).

Tabela 9: Diagnósticos de enfermagem segundo o domínio "conforto",

fatores relacionados e características definidoras, Goiânia, 2006.

\begin{tabular}{|c|c|c|c|c|c|}
\hline Categoria diagnóstica & $\mathbf{n}$ & Fatores relacionados & $n$ & Características definidoras & $\mathbf{n}$ \\
\hline & & & & Relato verbal (de dor) & 01 \\
\hline Dor crônica $\left(P_{2}\right)$ & 01 & Agente lesivo (físico). & 01 & & 01 \\
\hline & & & & $\begin{array}{c}\text { Posição antálgica para evitar a } \\
\text { dor }\end{array}$ & 01 \\
\hline Dor aguda $\left(P_{1}\right.$ e $\left.P_{7}\right)$ & 02 & $\begin{array}{l}\text { Agente lesivo (biológico). } \\
\text { Agente lesivo (físico). }\end{array}$ & $\begin{array}{l}01 \\
01\end{array}$ & Relato verbal (de dor) & 02 \\
\hline
\end{tabular}

Dor aguda foi identificada em dois participantes, sendo, em um deles relacionada a agente lesivo físico (prótese mal adaptada) e em outro agente lesivo biológico (hipóxia tecidual e neuropatia diabética), associada a relato verbal (Tabela 9).

Os problemas neuropáticos produzem, simultaneamente, dor, sob forma de queimação e ardor, diminuição da sensibilidade, fraqueza nas pernas e pés, junto a uma ausência dos reflexos do joelho e pés ${ }^{(10)}$.

\section{CONCLUSÃO}

Este estudo permitiu identificar um conjunto de 11 diagnósticos de enfermagem que ocorreram em todas as sete pessoas com diabetes tipo 2 que participaram da pesquisa: controle ineficaz do regime terapêutico; comportamento de busca de saúde risco para perfusão tissular cardíaca, renal, periférica e/ou cerebral ineficaz; percepção sensorial perturbada (visual); conhecimento deficiente sobre alimentação; cuidado com as extremidades; complicações da doença e prática de exercícios físicos; disposição para conhecimento aumentado acerca do diabetes mellitus tipo 2 e do regime terapêutico; risco de infecção; risco de lesão micro e macrovascular; risco para quedas e risco para integridade da pele prejudicada.

Outros diagnósticos encontrados na maioria dos participantes foram: nutrição desequilibrada: mais do que o corpo necessita (6 participantes), disfunção sexual (5 participantes) e percepção sensorial tátil (nos pés) perturbada (4 participantes).

Outros 10 diagnósticos foram encontrados, como peculiaridades de alguns participantes: dentição prejudicada, dor aguda, dor crônica, adaptação prejudicada, ansiedade, enfrentamento ineficaz, enfrentamento familiar comprometido, percepção sensorial perturbada (gustativa e olfativa), déficit de autocuidado com as unhas dos pés e intolerância à atividade.

Alguns destes diagnósticos de enfermagem estão diretamente ligados ao fato destas pessoas apresentarem diabetes, outros se referem a condições independentes desta patologia e outros se representam como resultantes do processo de envelhecimento.

É importante frisar que este panorama de diagnósticos foi identificado mediante abordagem baseada no Modelo de Orem. Se fossem empregados outros modelos teóricos, os diagnósticos identificados poderiam ser diferentes. Contudo, apesar disto e do pequeno número de participantes neste estudo, os resultados encontrados nos fornecem bases orientadoras para o planejamento do atendimento não só de enfermagem, mas de saúde, a pessoas com diabetes.

Estudos semelhantes a este, em que se identifiquem os condicionantes básicos do 
autocuidado e diagnósticos de enfermagem presentes em outros grupos de diabéticos, de diferentes áreas geográficas, seriam úteis para a elaboração de um mapa que pudesse ser utilizado não só no ensino, durante a formação de recursos humanos de enfermagem, bem como nas ações de educação permanente em saúde.

\section{REFERÊNCI AS}

1. Ministério da saúde; Secretaria de Atenção à Saúde. Departamento de Atenção Básica. Diabetes Mellitus. Cadernos de Atenção Básica n. 16. Brasília (Brasil): Ministério da Saúde, Secretaria de Atenção à Saúde; 2006.

2. Sociedade Brasileira de Diabetes. Consenso Brasileiro sobre Diabetes 2002: diagnóstico e classificação do diabetes melito e tratamento do diabetes melito do tipo 2. Rio de Janeiro (Brasil): SBD; 2003.

3. Sociedade Brasileira de Diabetes. Atualização Brasileira sobre Diabetes/Sociedade Brasileira de Diabetes. Rio de Janeiro (Brasil): Diagraphic; 2006.

4. Grupo de trabalho internacional sobre pé diabético. Consenso Internacional sobre Pé Diabético/ publicado sob direção de Hermelinda Cordeiro Pedrosa. Brasília (Brasil): Secretaria de Estado de Saúde do Distrito Federal; 2001.

5. Costa AA, Almeida Neto JS. Manual de Diabetes: educação, alimentação, medicamentos, atividades físicas. 4a edição. São Paulo: Sarvier; 2004.

6. North American Nursing Diagnosis Association. Diagnósticos de Enfermagem da NANDA: definições e classificação - 2005-2006/ organizado por North American Nursing Association. Porto Alegre (Brasil): Artmed; 2006.

7. Orem ED. Modelo de Orem - Conceptos de enfermería em la práctica. Barcelona: Masson I tália Editori; 1993.

8. Vasconcelos LB de, Adorno J, Barbosa MA, Sousa JT. Consulta de enfermagem como oportunidade de conscientização em diabetes. Revista Eletrônica de Enfermagem [serial on the internet]. 2000 [cited 2005 Mai 16];2(2). Available from: http://www. fen. ufg. br/revista/revista2_2/diabete.html.

9. Péres DS, franco LJ, Santos MA. Comportamento alimentar em mulheres portadoras de diabetes tipo 2. Revista de Saúde Pública. 2006; 40(2): 310-7.

10. Pace AE, Nunes PD, Ochoa-Vigo K. O conhecimento dos familiares acerca da problemática do portador de diabetes mellitus. Revista Latino-Americana de Enfermagem. 2003; 11(3): 312-9.

11. Araújo SSC, Feire DBL, Padilha DMP, Baldisserotto J. Suporte social, promoção de saúde e saúde bucal na população idosa no Brasil. Interface. 2006; 10(19): 203-16.

12. Almeida MF, barata RB, Montero CV, Silva ZP. Prevalência de doenças crônicas autoreferidas e utilização de serviços de saúde, PNAD/1998, Brasil. Ciência \& Saúde Coletiva. 2002; 7(4): 743-56.

13. Sartorelli DS, Franco LJ, Cardoso MA. Intervenção nutricional e prevenção primária do diabetes mellitus tipo 2: uma revisão sistemática. Cadernos de Saúde Pública. 2006; 22(1): 7-18.

14. Campos MAG, Pedroso ERP, Lamounier JA, Colosimo EA, Abrantes MM. Estado nutricional e fatores associados em idosos. Revista da Associação Médica Brasileira. 2006;52(4):21214.

15. Smeltzer SC, Bare BG. Tratado de enfermagem médico-cirúrgica. 10ạ edição. Rio de Janeiro: Guanabara Koogan; 2005.

16. Bosco A, Lerário AC, Soriano D, Santos RF, Massote $P$, Galvão $D$, et al. Retinopatia diabética. Arquivos Brasileiros de Endocrinologia \& Metabologia. 2005; 49(2):217-27.

17. Gazzinelli MF, Gazzinelli A, Reis DC, Penna CMM. Educação em saúde: conhecimentos, representações sociais e experiências da doença. Cadernos de Saúde Pública. 2005; 21(1): 200-6.

18. Potter PA. Semiologia em Enfermagem. 4a edição. Rio de Janeiro: Reichmann \& Affonso Editores; 2002.

19. Almeida NAM, Silva LA, Araújo NM. Conhecimento de acadêmicas de enfermagem sobre disfunções sexuais femininas. Revista Eletrônica de Enfermagem [Internet]. 2005 [cited 2006 sep 08];7(2):47-138. Available from:

http://www.fen.ufg. br/revista/revista7_2/pdf/O RIGINAL_01.pdf. 
Milhomem ACM, Mantelli FF, Lima GAV, Bachion MM, Munari DB.Diagnósticos de enfermagem identificados em pessoas com diabetes tipo 2 mediante abordagem baseada no Modelo de Orem. Revista Eletrônica de Enfermagem [Internet]. 2008; 10(2):321-336. Available from: http://www.fen.ufg.br/revista/v10/n2/v10n2a04.htm

20. Amaral FMF, Ramos PGA, Ferreira SRG.

Estudo da freqüência de cárie e fatores associados no diabetes mellitus tipo 1. Arquivos

Brasileiros de Endocrinologia \& Metabologia.

2006;50(3):515-22.

Artigo recebido em 05.02.07

Aprovado para publicação em 30.06.08 